\title{
UCRL-TR-227709
}

LA W REN CE LIVERMORE N A TIONAL LABORATORY

High Resolution Imaging of Satellites with Ground-Based 10-m Astronomical Telescopes

C. Marois

February 2, 2007 
This document was prepared as an account of work sponsored by an agency of the United States government. Neither the United States government nor Lawrence Livermore National Security, LLC, nor any of their employees makes any warranty, expressed or implied, or assumes any legal liability or responsibility for the accuracy, completeness, or usefulness of any information, apparatus, product, or process disclosed, or represents that its use would not infringe privately owned rights. Reference herein to any specific commercial product, process, or service by trade name, trademark, manufacturer, or otherwise does not necessarily constitute or imply its endorsement, recommendation, or favoring by the United States government or Lawrence Livermore National Security, LLC. The views and opinions of authors expressed herein do not necessarily state or reflect those of the United States government or Lawrence Livermore National Security, LLC, and shall not be used for advertising or product endorsement purposes.

This work performed under the auspices of the U.S. Department of Energy by Lawrence Livermore National Laboratory under Contract DE-AC52-07NA27344. 


\title{
High Resolution Imaging of Satellites with Ground-Based 10-m Astronomical Telescopes
}

\author{
Christian Marois \\ cmarois@igpp.ucllnl.org \\ Lawrence Livermore National Laboratory, IGPP, L-413, \\ Livermore, CA, 94550
}

Received __ ; accepted _ 


\begin{abstract}
High resolution imaging of artificial satellites can play an important role in current and future space endeavors. One such use is acquiring detailed images that can be used to identify or confirm damage and aid repair plans. It is shown that a 10-m astronomical telescope equipped with an adaptive optics system (AO) to correct for atmospheric turbulence using a natural guide star can acquire high resolution images of satellites in low-orbits using a fast shutter and a near-infrared camera even if the telescope is not capable of tracking satellites. With the telescope pointing towards the satellite projected orbit and less than 30 arcsec away from a guide star, multiple images of the satellite are acquired on the detector using the fast shutter. Images can then be shifted and coadded by post processing to increase the satellite signal to noise ratio. Using the Keck telescope typical Strehl ratio and anisoplanatism angle as well as a simple diffusion/reflection model for a satellite $400 \mathrm{~km}$ away observed near Zenith at sunset or sunrise, it is expected that such system will produced $>10 \sigma \mathrm{K}$-band images at a resolution of $10 \mathrm{~cm}$ inside a 60 arcsec diameter field of view. If implemented, such camera could deliver the highest resolution satellite images ever acquired from the ground.
\end{abstract}

Subject headings: High resolution imaging

Suggested running page header: Satellites Imaging

\title{
1. Introduction
}

Satellite imaging has long been an interest for both civil and military communities. Following the February 2003 Columbia incident, it was acknowledged that high resolution 
imaging might have found the shuttle wing anomaly and is now recommended for each shuttle flight (CAIB 2003). In the context of the SS return to flight, the ISS completion and future missions, developing high resolution imaging capability is crucial to adequately support these endeavors.

Ground-based satellite imaging is limited by atmospheric turbulence that degrades the resolution and by fast apparent satellite motion that makes them impossible to track with conventional telescopes. Some images have been acquired with amateur 0.3-m telescopes with some success but these are limited by the atmospheric turbulence and the small aperture (Dantowitz 1996). Development of specialized telescopes that can track satellites, like the Maui AEOS and Star Fire optical range observatories, have obtained good results, but are also limited by the 1 to 3.6-m telescope apertures and the atmospheric turbulence. These telescopes obtain diffraction limited images by acquiring short exposure images and combining them using the multi-frame blind deconvolution technique (Schulz 1993). Even if these observatories have high order AO systems and can track the SS and ISS, it is very difficult to do AO-corrections on such large resolved objects.

To adequately monitor satellites, the SS, the ISS and future space craft on successive orbits and from multiple sites, an easy to use and easy to implement high resolution imaging system is required. If the fast apparent motion of these objects can be overcome, higher resolution images can be obtained using existing 10-m astronomical telescopes and their adaptive optics (AO) systems even if these observatories cannot track satellites. The geographical location of giant telescopes across the globe, is ideal for such monitoring. Additionally, since observations are made at sunset and sunrise to optimize satellite illumination, the impact on the scheduled night time astronomical observations is minimal.

This paper presents the concept of a fast shutter and near infrared camera that could be used on existing 10-m class telescopes to overcome the fast apparent motion of 
low-orbiting satellites. Diffraction limited images are obtained with an AO system using a natural guide star chosen close to the projected satellite orbit. The imaging concept is first presented, followed by predicted and simulated performances. Daily monitoring is then discussed. Finally, future possibilities with current 10-m telescopes and next generation 30 to $100-\mathrm{m}$ telescopes are mentioned.

\section{High Resolution Imaging of Satellites with Ground-based 10-m Telescopes}

Satellite typical sizes range from a meter to 100-m length. If we assumed that these satellites are in low orbit, at $350 \mathrm{~km}$ like the ISS, they have an apparent angular size of $0.5^{\prime \prime}$ up to $50^{\prime \prime}\left(15^{\prime \prime} \& 50^{\prime \prime}\right.$ for respectively the SS and the ISS). Since they orbit the Earth in 90 minutes, seen from the surface of the Earth, they have an angular speed, at zenith, of $3800^{\prime \prime} / \mathrm{s}$, thus impossible to track with astronomical telescopes. For example, the Keck 10-m telescope can slew up to $3600^{\prime \prime} \mathrm{s}^{-1}$ but can only track (open loop) up to $6^{\prime \prime} \mathrm{s}^{-1}$.

One can overcome the angular speed problem by using a fast shutter to acquire multiple satellite images while the satellite crosses the camera field of view (FOV). Diffraction limited images are acquired by using a natural guide star to close the AO loop. The guide star is selected several arcsec away from the projected satellite orbit ${ }^{1}$, so that the satellite goes through the FOV without occulting the guide star, and close to zenith to maximize apparent size of the satellite and to improve atmospheric wavefront conditions. K-band ( $2.2 \mu \mathrm{m}$ with $0.3 \mu \mathrm{m}$ bandpass) imaging is chosen to maximize Strehl ratio and the anisoplanatism angle. Finally, the Sun-satellite-observatory angle needs to be minimized to offer the maximal satellite illumination. The fast shutter is used to obtain multiple

\footnotetext{
${ }^{1}$ Typical $1 \sigma$ ISS orbit predictions are accurate to less than $30 \mathrm{~m}$ (Jonathan K. Weaver, private communication), or less than half the Keck K-band anisoplanatism angle.
} 
images of the satellite while it goes through the anisoplanatism angle in a single detector readout. The fast shutter could be a rotating slit in the image plane that scan the FOV every several microseconds or a fast pupil shutter like a Q-switch. See Fig. 1 for a typical implementation. For the rest of the paper, observations are assumed to be made from the Keck observatory, showing a typical K-band (2.2 $\mu \mathrm{m}$ with $0.3 \mu \mathrm{m}$ bandpass) on-axis Strehl ratio of the order of 0.5 and anisoplanatism angle of $60^{\prime \prime}$ diameter. The resolution power of AO-corrected 10-m class astronomical telescopes is typically $0.045^{\prime \prime}$ in K-band, thus corresponding to $10 \mathrm{~cm}$ at $400 \mathrm{~km}$.

\section{Predicted Performances}

The number of detected photons per $\lambda / D$ per second is now estimated. Due to the very complex problem of estimating a satellite's brightness, a simple isotropic diffusion model is considered here. It is expected that different parts of the satellite can be fainter or brighter than this simple model depending of the surface reflectivity and Sun-satellite-observatory geometry. The Sun's spectrum is approximated by a Planck function of flux density $f=4 \times 10^{5} \mathrm{erg} / \mathrm{cm}^{2} / \mathrm{s} / \AA$ at $2.2 \mu \mathrm{m}$. The flux density at $1 \mathrm{AU}$ is found by integrating over the surface of the Sun (radius of $R_{\text {Sun }}$ ), and dividing by the surface of a 1 AU radius sphere. The flux density at the telescope pupil is obtained by integrating the flux $f$ for a typical $R_{\text {Surf }}=5 \mathrm{~cm}$ radius surface situated at $R_{\text {Orb }}=400 \mathrm{~km}$ from an observatory and assuming an isotropic photon diffusion with a reflection coefficient $r=80 \%$ at $2.2 \mu \mathrm{m}$. Integrating for the telescope diameter $D$, total instrument transmission and detector quantum efficiency $t=0.2$, encircled energy $e=0.5$ inside one $\lambda / D$ and a filter bandpass $\Delta \lambda=0.31 \mu \mathrm{m}$, we obtain $1.4 \times 10^{7}$ electrons per $\lambda / D$ per second. Eq. 1 summarizes the integrated flux (electrons per second) $f_{\text {Dec }}$ per $\lambda / D$ from a satellite. 


$$
f_{\text {Dec }}=10^{-7} \frac{\pi}{2} \text { fret } \frac{\lambda \Delta \lambda}{h c}\left(\frac{R_{\text {Sun }}}{1 \mathrm{AU}}\right)^{2}\left(\frac{R_{\text {Surf }}}{R_{\text {Orb }}}\right)^{2}\left(\frac{D}{2}\right)^{2}
$$

where $c$ is the speed of light and $h$ is the Planck constant. To minimize the satellite motion during an exposure, an exposure time limiting the object's motion to one $\lambda / D$ is chosen. For a $\lambda / D$ of $0.045^{\prime \prime}$ and a satellite traveling at $3800^{\prime \prime} / \mathrm{s}$, an exposure time of $12 \mu \mathrm{s}$ is found; thus, from Eq. 1, 170 electrons per $\lambda / D$ are detected. Since satellite angular sizes are usually smaller than the anisoplanatism angle, multiple images can be acquired and combined to increase its $\mathrm{S} / \mathrm{N}$. The shutter frequency is calculated with the satellite angular dimension and its speed. For the SS traveling at $3800^{\prime \prime} / \mathrm{s}$ and having an angular size of $15^{\prime \prime}$, an image every $4 \mathrm{~ms}$ is required while the ISS (50" diameter) traveling at the same speed requires an image every $13 \mathrm{~ms}$. To get theses short exposure times at different frame rates, the shutter will need to be optimized for each observations.

From the previous calculation, it was found that each $\lambda / D$ area has in average 170 electrons, thus a corresponding photon noise of $\sqrt{170}=13$ electrons RMS. To optimize the $\mathrm{S} / \mathrm{N}$ ratio, it is important to minimize the thermal background so that it is smaller than this value. A typical exposure will be of the order of $30 \mathrm{~s}$ to make sure that the satellite is acquired. Using the estimated K-band sky background at Keck as an upper limit (12.6 mag per $\operatorname{arcsec}^{2}$ at night, or approximately 200 electrons per $\lambda / D$ per second), for a 30 s exposure, we obtain 6000 electrons per $\lambda / D$ and 80 electrons RMS, significantly larger than the photon noise per $\lambda / D$ from the satellites. For the same $30 \mathrm{~s}$ exposure with a cryogenic shutter set to acquire $12 \mu$ s exposures every $4 \mathrm{~ms}$, we obtain 4 electrons RMS. A low emissivity or cryogenic shutter may thus be required. At sunset and sunrise, a sky background of up to 100 electrons (10 electrons RMS) can be tolerated without significantly reducing the final $\mathrm{S} / \mathrm{N}$. Read noise can be reduced from a typical 18 electrons RMS to 6 electrons RMS by using 9-9 multiple Fowler sampling (Fowler \& Gatley 1990). Using this 
setup, the dominant noise will be the photon noise inside one $\lambda / D$, typically $\sim 10$ electrons, thus producing images with $\mathrm{S} / \mathrm{N}$ of $\sim 10$.

Finally, since low-orbit satellites are not at infinity but at $\sim 400 \mathrm{~km}$ for the ISS and $\mathrm{SS}$, the camera focus will need to be adjusted. If the camera is set at infinity, an object at $400 \mathrm{~km}$ will suffer from 9 microns RMS of focus aberration. At $300 \mathrm{~km}$ or $500 \mathrm{~km}$, we obtain 7 and 12 microns RMS respectively. It is thus necessary to have a camera that can have its focus optimized to produce sharp images.

\section{Simulated Performances}

Simulated images are now calculated to demonstrate the technique. A turbulent phase screen is first generated using a power law spectrum with index value of -11/3 (Noll 1976) having a variance of $1 \operatorname{rad}^{2}\left(\mathrm{D} / \mathrm{r}_{0}\right)^{5 / 3}$. Simulations were produced for a 10 -m diameter telescope and $\mathrm{r}_{0}$ of $1.3-\mathrm{m}$ at $\mathrm{K}$-band. To produce the anisoplanatism angle consistent to what is observed at the telescope, the turbulence layer is chosen to be $3 \mathrm{~km}$ above the observatory. The AO system is conjugated at the pupil, as the current Keck 2 AO system. The AO-corrected wavefront is obtained by leveling the phase error power spectrum for spatial frequencies smaller than twice the actuator's frequency. To estimate the simulated anisoplanatism, off-axis PSFs are calculated by subtracting the on-axis wavefront correction from the shifted uncorrected atmospheric phase screen. Fig. 2 shows the Strehl ratio as a function of angular separation for our simulations. Our simulations fit well the observed

K-band Strehl ratio as a function of angular separation observed for the Keck observatory (see http://www2.keck.hawaii.edu/optics/ngsao/ for typical Keck AO performances).

Visible images of the ISS, SS and an astronaut from the NASA website are converted to grayscale and properly scaled to the correct number of pixels assuming a distance of 
$400 \mathrm{~km}$ from the telescope (30 degrees from zenith for an orbital altitude of $350 \mathrm{~km}$ ). Even if these images were acquired in the visible, they are chosen here for K-band images for the sake of the simulation. Flux were calibrated so that the brightest part of each object gets twice the estimated 170 photons per $\lambda / D$ value so that the average brightness be close to our prediction. Since the objects are not at infinity, similarly to a laser guide star, wavefront correction will suffer from a cone effect (Foy \& Labeyrie 1985). Since these objects are at higher altitude compared to a sodium laser guide stars (Thompson \& Gardner 1987) (400 km instead of $70 \mathrm{~km}$ altitude), the effect will be less severe. Off-axis PSF for $400 \mathrm{~km}$ satellites are thus produced by properly shifting the uncorrected atmospheric wavefront, by spatially scaling the wavefront errors to account for the cone effect and by subtracting the on-axis AO corrections. A focal plane rotating slit is assumed for the fast shutter. Since the satellite is moving while the slit scans the focal plane, satellites will be sheared. It is assumed here that the camera is rotated to align the projected satellite orbit on the detector $\mathrm{X}$-axis. Finally, images are convolved by a one $\lambda / D$ motion blur to simulate satellite motion during exposures. Fig. 3 shows a typical raw $4 \mathrm{~K} \times 4 \mathrm{~K} \mathrm{~K}$-band image. Since the ISS is quite large, it is impossible to get more than one image inside the anisoplanatism angle on existing 10-m telescopes AO systems. Since multiple images of the SS and of the astronaut are acquired, they are shifted and coadded to increase their S/N. Fig. 4 and Fig. 5 show the final combined image of the ISS, the SS and the astronaut. Note that, in Fig. 5, the space shuttle name is clearly visible as well as details of the astronaut.

Fig. 6 shows the SS with and without photon, sky and read noises as well as a simulation with $10 \%$ of the predicted flux. It is clear that the image quality is photon noise limited. Increasing the instrument throughput would definitely results in better images. For example, since SS images are acquired every $4 \mathrm{~ms}$ with an exposure time of only $12 \mu \mathrm{s}$, if a fast tip/tilt system was used instead of a fast shutter to move the FOV across the detector every $12 \mu \mathrm{s}$, numerous images could be acquired (up to 1,500 SS images if a big $12 \mathrm{k} \times 12 \mathrm{k}$ 
detector is used) instead of only a few if we wait for the SS to move by one of its diameter before acquiring a new image. $\mathrm{S} / \mathrm{N}$ ratio up to 22 times better could be achieved by this approach. To get such fast FOV motion, a $83 \mathrm{KHz}$ tip/tilt system is needed. If such system is implemented, a cold field stop having a radius equal the anisoplanatism angle is required to avoid an over luminous sky background.

\section{NGS Guide Star Frequency}

To be able to acquire high quality satellite images, a natural guide star needs to be selected not too far from the satellite projected orbit and as close as possible to zenith. For Keck, having an anisoplanatism angle of 60 arcsec diameter in K-band, a maximum

distance of 30 arcsec is chosen. The Keck LGS IDL guide star program and the Strasbourg Simbad database were used to estimate the frequency of natural guide star for Mauna Kea. Every 60 arcsec position between -10 and 5 degrees of declination (60-75 degrees altitude at Mauna Kea) for every integer value of right ascention were checked for guide stars within 30 arcsec brighter than 12th magnitude in R-band. Typically, one or more 10th magnitude guide stars at less than 10 arcsec are found per 15 degree projected orbit. Having a guide star available for a given projected orbit is thus not problematic.

\section{Daily Monitoring}

Astronomical telescopes offer a unique way to do daily monitoring of satellites since astronomical images are taken after sunsets and before sunrises. Observations would thus have a minimal impact on astronomical observations as long as changing instrument is made easy. The use of a deployable pick up mirror under the AO system would be ideal. Since a large number of 8-10 m telescopes are already built and almost all of them are 
already equipped with AO systems, a multi site, multi orbit monitoring is thus feasible. The possibility of using 4-m class telescopes, albeit losing a factor of 2 in resolution, would also be interesting. The Heavens-above web site (http://www.heavens-above.com/) was used to estimate the frequency of higher than 45 degrees altitude events for a given observatory. As expected, the best place to acquire a given satellite is where the latitude of the observatory is equal to the satellite orbit inclination. Telescope sites at higher latitudes (or lower in the southern hemisphere) are thus more favorable for the ISS since it has a 45 degree orbit inclination (one event every few days for the Lick observatory), while the Mauna Kea observatories are better for satellites having $\sim 20$ degree orbit inclination (like the Hubble Space Telescope). If a servicing mission is sent to HST, Mauna Kea observatories could acquire the SS and HST every few days.

\section{Future Possibilities}

Future astronomical facilities upgrades will also offer interesting possibilities. A multi-conjugate natural guide star AO system (MCAO) could offer a bigger anisoplanatism angle, thus enabling an increased number of acquired image and better $\mathrm{S} / \mathrm{N}$, but using such approach will probably requires artificial off-axis laser guide stars to obtain good sky coverage. Another possibility is to build a multi-wavelength camera to acquire simultaneously JHK images, thus increasing the number of images and spectral coverage. Future $30-\mathrm{m}$ and 100-m telescopes will offer higher resolution images $(3$ to $1 \mathrm{~cm}$ ) on a smaller field of view unless a MCAO is used. These new observatories could be used to track satellites on higher orbits, possibly as far as the geosynchronous orbit (3 to $1 \mathrm{~m}$ resolution). High resolution images of near-Earth fast moving meteors would also be feasible. 


\section{Conclusion}

It was shown that a relatively simple cryogenic near infrared camera mounted under an existing $\mathrm{AO}$ system of a 10-m astronomical telescope can achieve $10 \mathrm{~cm}$ resolution at K-band for low orbiting satellites. The large anisoplanatism angle and the usually small satellite size mean that multiple images can be acquired inside the anisoplanatism angle and can be combined to increase the satellite $\mathrm{S} / \mathrm{N}$. Typical $\mathrm{S} / \mathrm{N}$ ratios per image is 10, up to 17 if 3 images are coadded. Instrument throughput can be further increased if a fast $83 \mathrm{KHz}$ tilt/tilt system and a big detector are used to produce numerous images while the satellite passes inside the anisoplanatism angle ( $\mathrm{SS} \mathrm{S} / \mathrm{N}$ gain up to $22 \mathrm{x}$ ). These images can be combined and used to support space mission and look for damage (e.g. SS tiles) or problems while deploying satellites. Typically one or more 10th magnitude natural guides stars can be found less than 10 arcsec away for each 15 degree projected orbit. Each observatory offers the best monitoring of satellites having an orbital inclination equal to their observatory latitude. Mauna Kea observatories are thus ideal for satellites with low inclination like HST. The availability of sunset/sunrise time of astronomical telescopes and their wide geographical distribution make them attracting to do daily monitoring. The diffraction limited images of large aperture astronomical telescopes offer a unique possibility to acquire high resolution satellite images that could play a crucial role in supporting current and future space endeavors.

The author would like to thank Bruce Macintosh, Brian Bauman, Lisa Poyneer, Étienne Artigau and David Lafrenière for discussions. This research was performed under the auspices of the US Department of Energy by the University of California, Lawrence Livermore National Laboratory under contract W-7405-ENG-48, and also supported in part by the National Science Foundation Science and Technology Center for Adaptive Optics, managed by the University of California at Santa Cruz under cooperative agreement AST 
98-76783. 


\section{REFERENCES}

Columbia Accident Investigation Board report 2003, volume 1, part 2, 76

Dantowitz, R. 1996, Sky \& Telescope, 86

Foy, R., Labeyrie, A. 1985, AAP, 152, L29

Fowler, A. M., \& Gatley, I. 1990, ApJ, 353, L33

Noll, R. J. 1976, OSAJ, 66, 207

Thompson, L. A., Gardner, C. S. 1987, Nature, 328, 229

T. J. Schulz 1993, JOSAA, 10, 1064 


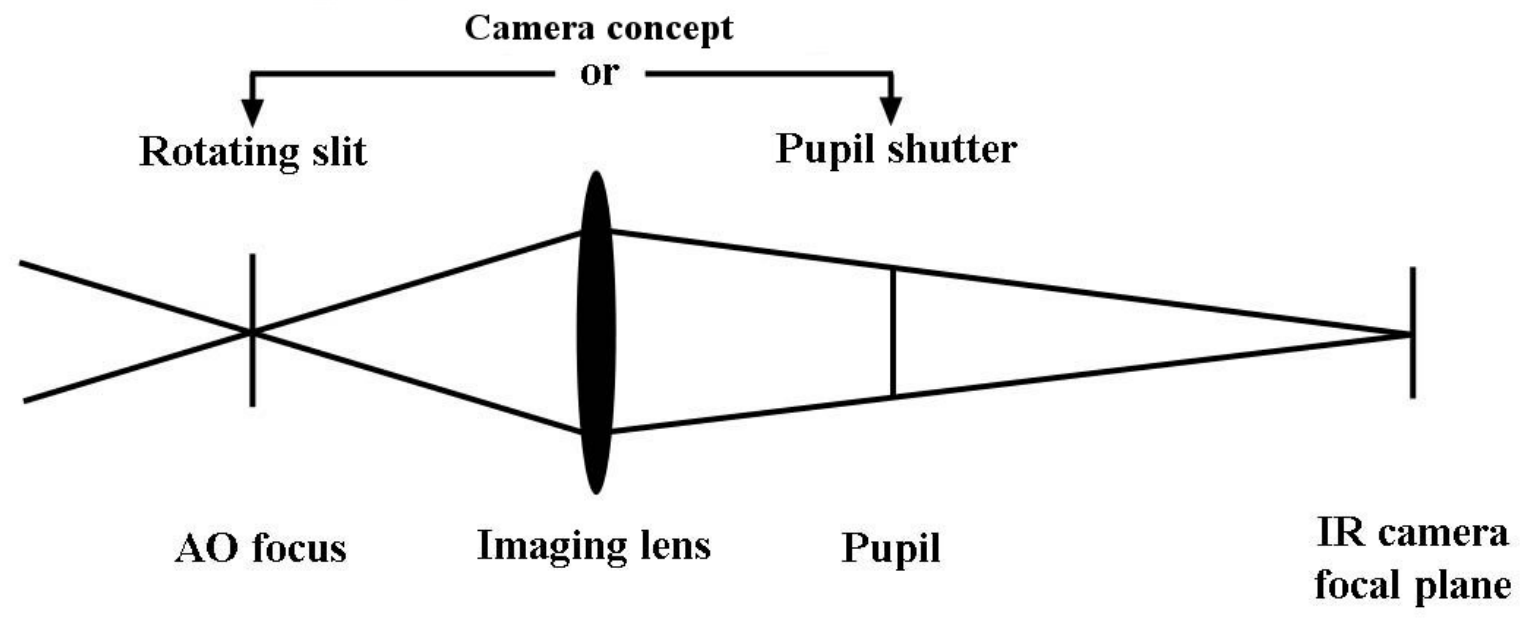

Fig. 1. - The near-infrared camera concept. Multiple satellite images are produced in a single detector readout by using a fast shutter. This shutter can be introduced at an AO focus plane (rotating slit) or in a reimaged pupil plane (Q-switch). A standard near-infrared camera is used to acquire the images. 


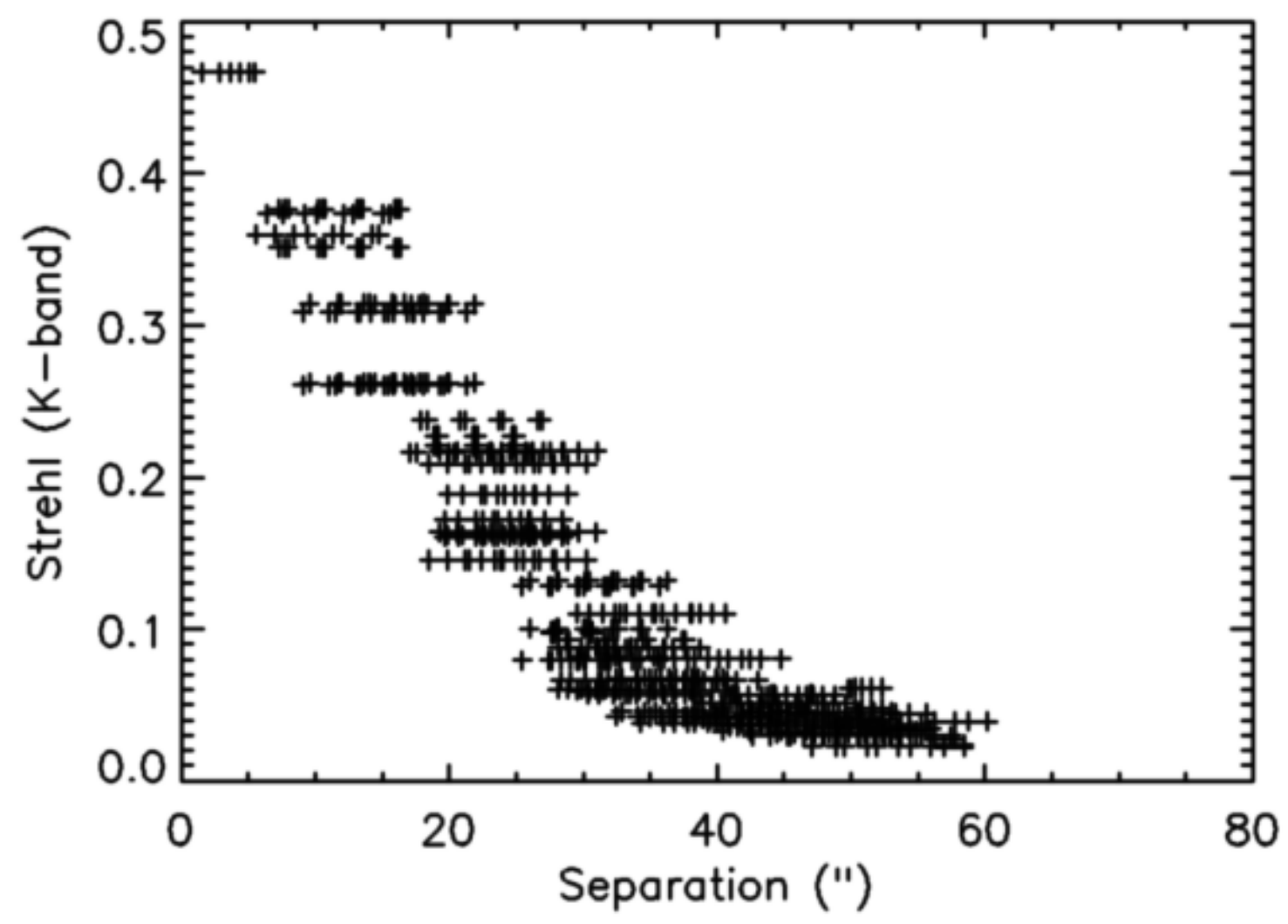

Fig. 2.- Simulated Strehl ratios (K-band) as a function of angular separation. 


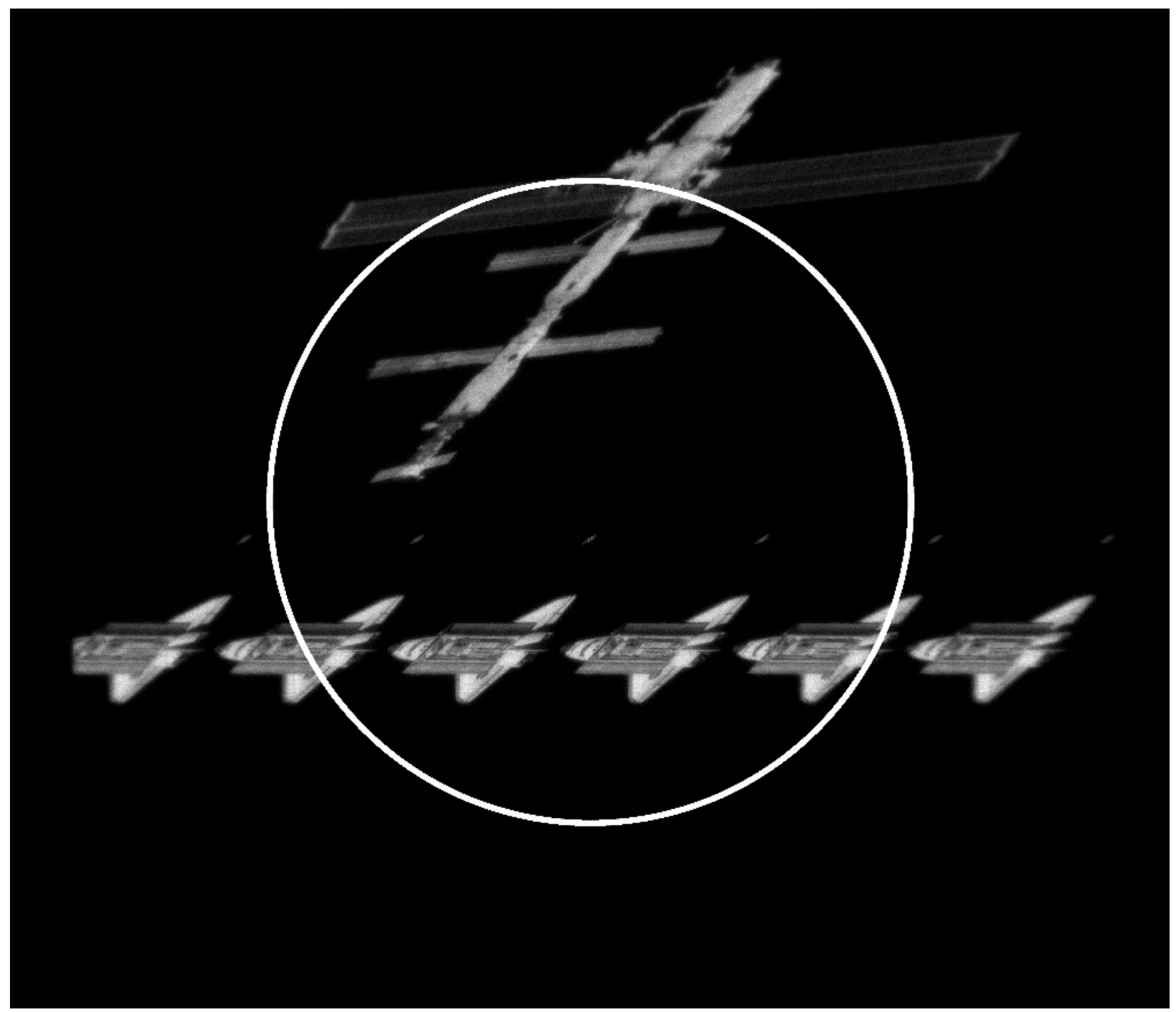

Fig. 3.- Simulated raw image. A focal plane rotating slit aligned with the $\mathrm{X}$-axis is assumed (focal slit scan from bottom to top). The SS, astronaut and ISS images are shown. Reference PSF was located between the SS and ISS 10 arcsec away from the SS (at the image center). The circle shows the Keck K-band anisoplanatism angle (30 arcsec radius). FOV is $1.8 \times 1.5$ arcmin. Unprocessed images from NASA. 


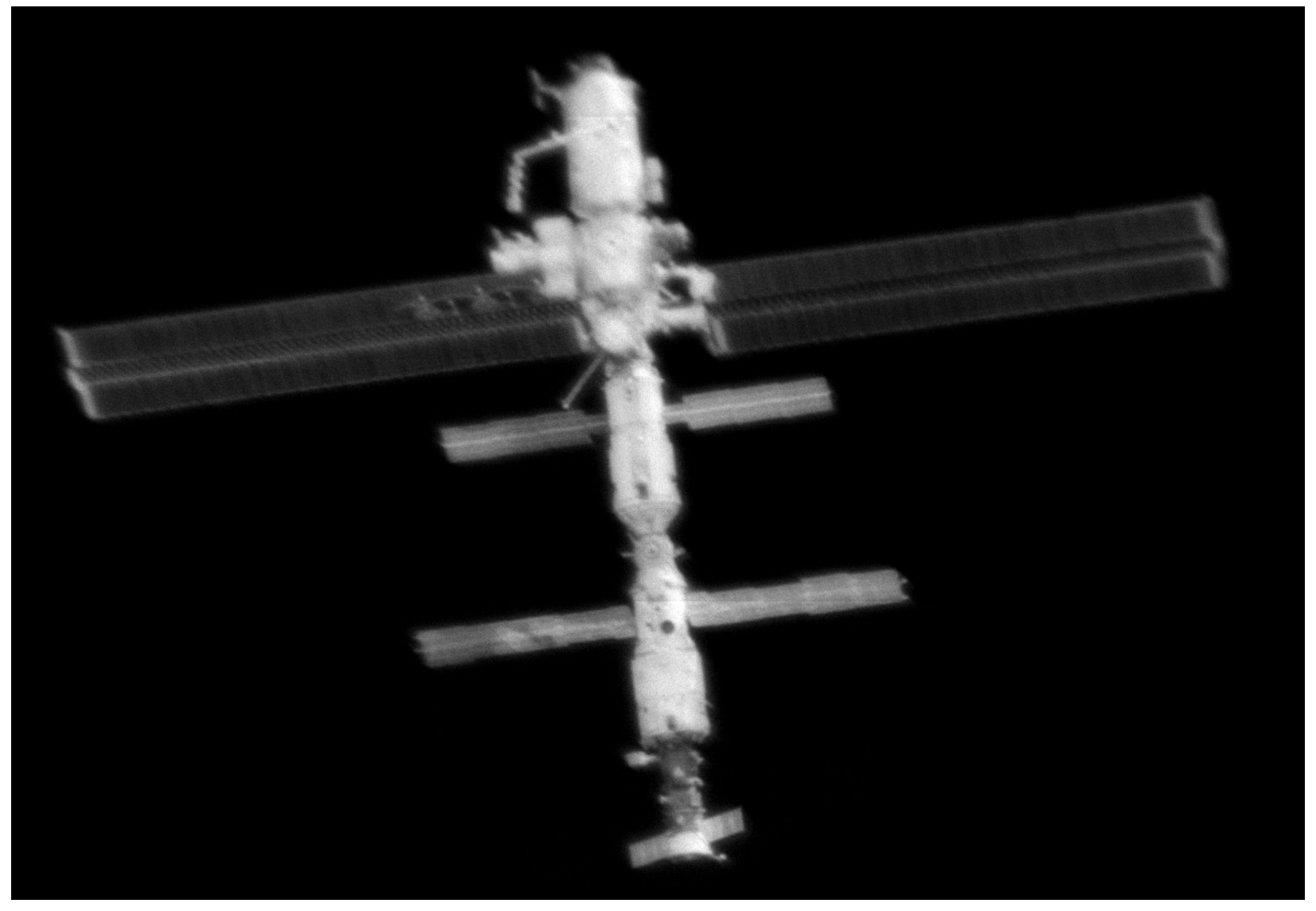

Fig. 4.- Same as Fig. 3 with the image shear corrected and zoomed on the ISS. Image has been convolved by a 2 pixel FWHM Gaussian to minimize photon noise. Unprocessed images from NASA. 

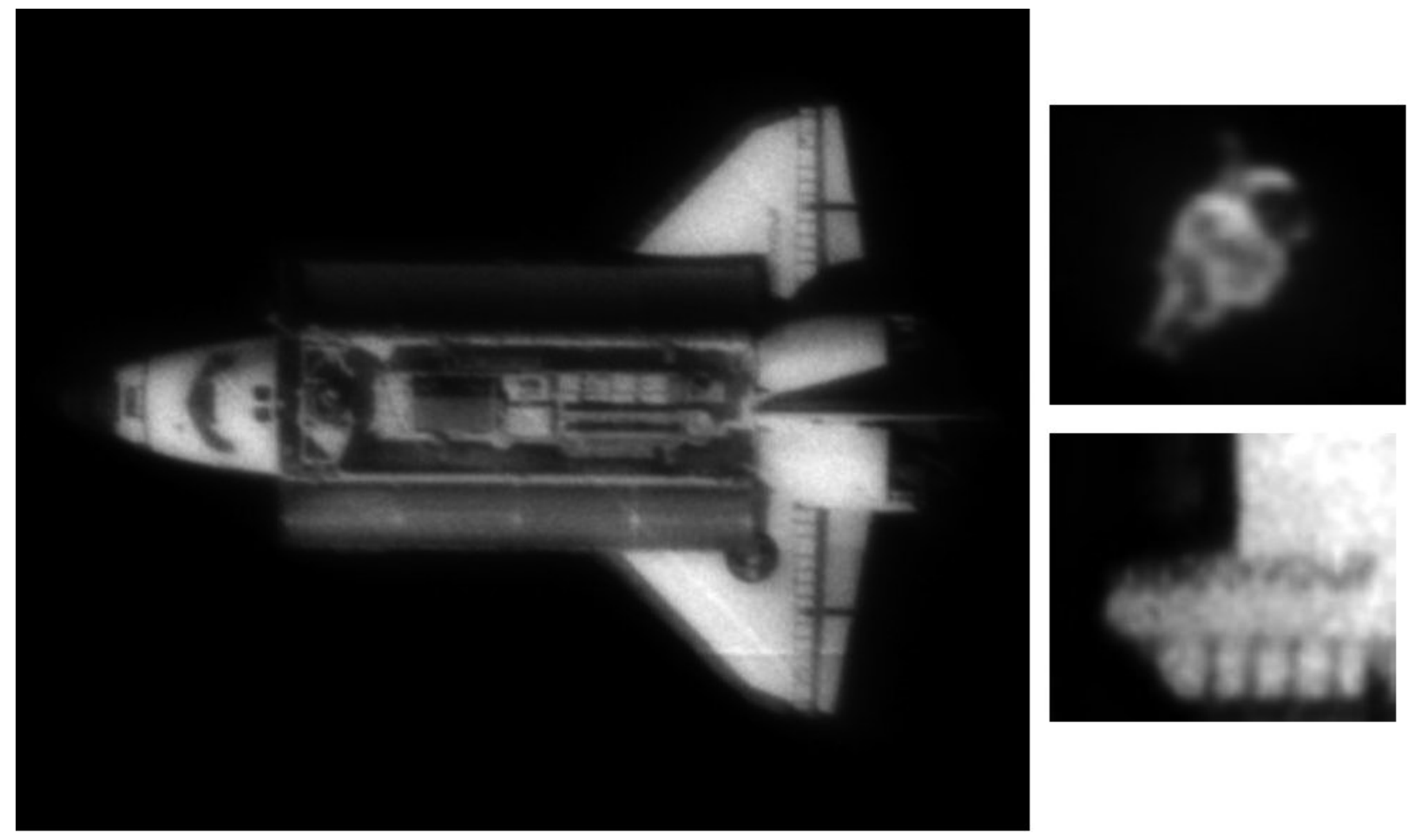

Fig. 5.- Same as Fig. 3, three individual shear corrected images were shifted and median combined to increase the $\mathrm{SS}$ and astronaut $\mathrm{S} / \mathrm{N}$ ratio. The image has been convolved by a 2 pixel FWHM Gaussian to minimize photon noise and sharpened with Gimp (http://www.gimp.org/). The astronaut and SS name are clearly visible. Unprocessed images from NASA. 

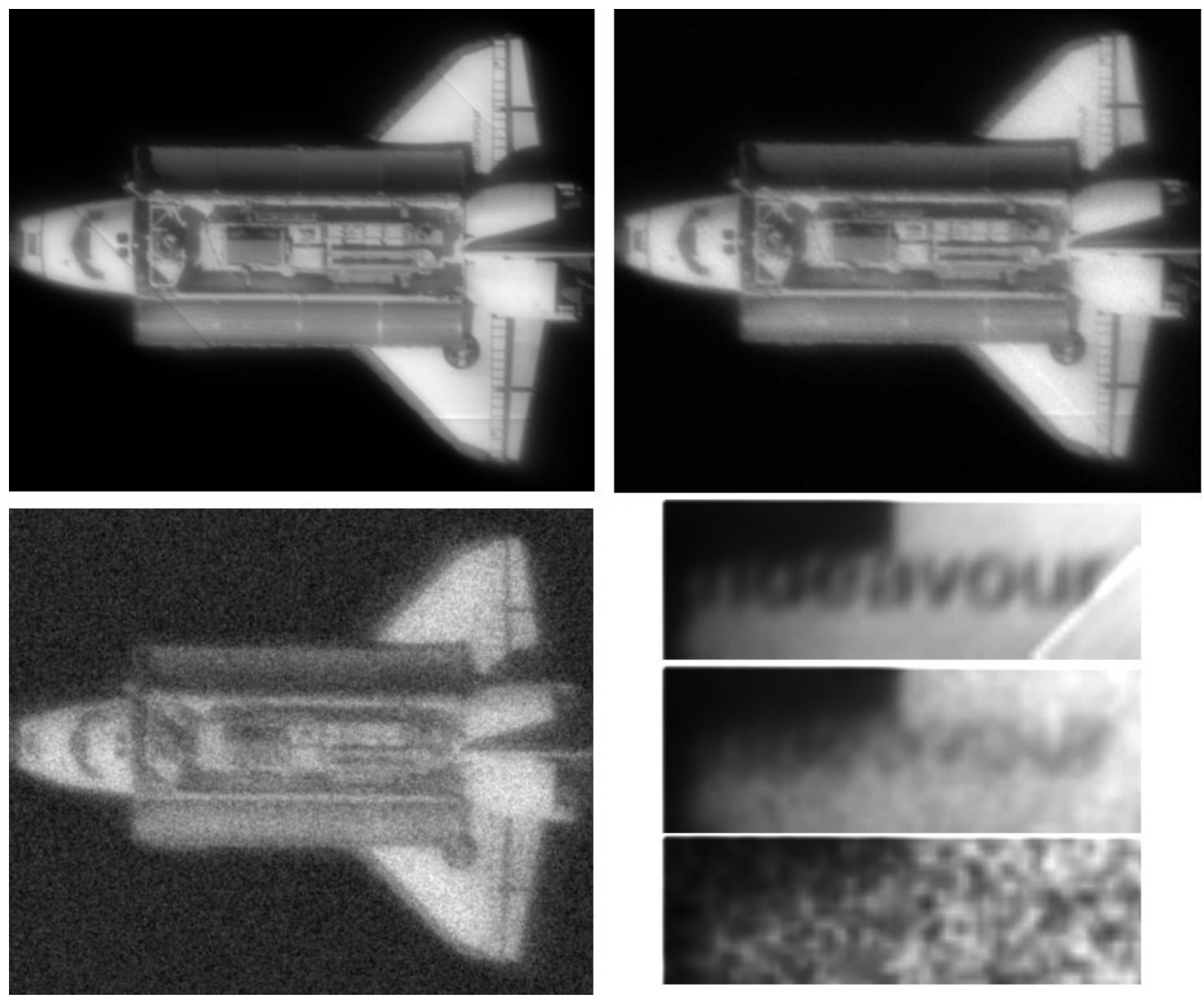

Fig. 6.- SS aberrated image as a function of flux intensity. Upper left panel shows the aberrated SS image without photon, sky and read noises, upper right panel shows the same SS image using the model estimated flux and with photon, sky and read noises included while the bottom left panel shows the same image but with $10 \%$ of the predicted flux. Both the upper right and bottom left panels are the average of three SS images and have been convolved by a 2 pixel FWHM Gaussian. Diagonal and horizontal lines in the upper left panel are numerical artifacts produced by the simulation. The bottom right panel shows three small SS wing sections centered on the SS name (from top to bottom, the SS name for the perfect $\mathrm{S} / \mathrm{N}$, model estimated and $10 \%$ of the model estimated value). Unprocessed images from NASA. 